\title{
Formation of Si nanocrystallites observed by in situ transmission electron microscopy and their effect on the enhancement of Er photoluminescence in $\mathrm{Er}$-doped $\mathrm{SiO}_{2}$
}

\author{
N. Fukata ${ }^{a), b), c) ~}$ \\ Advanced Electronic Materials Center, National Institute for Materials Science, 1-1 Namiki, \\ Tsukuba 305-0044, Japan
}

H. Morihiro, R. Shirakawa, and K. Murakami ${ }^{\mathrm{b})}$

Institute of Applied Physics, University of Tsukuba, Tsukuba 305-8573, Japan

M. Mitome and Y. Bando

Nanoscale Materials Center, National Institute for Materials Science, 1-1 Namiki, Tsukuba 305-0044, Japan

(Received 15 August 2007; accepted 1 October 2007; published online 12 December 2007)

\begin{abstract}
The formation of $\mathrm{Si}$ nanocrystallites $(\mathrm{nc}-\mathrm{Si})$ in erbium $(\mathrm{Er})$-dispersed $\mathrm{SiO}_{x}(x \leqslant 2)$ films was investigated by in situ annealing while performing transmission electron microscopy measurements. The correlation between the formation of nc-Si and Er ion emissions was also comprehensively investigated by photoluminescence and electron spin resonance measurements. The results showed that the formation of nano-Si region with the suitable size is important for enhancement of Er ion emission. (C) 2007 American Institute of Physics. [DOI: 10.1063/1.2817639]
\end{abstract}

\section{INTRODUCTION}

The sensitization of Er ion emission $(\lambda=1.54 \mu \mathrm{m})$ by host materials, such as crystalline $\mathrm{Si}(c-\mathrm{Si}),{ }^{1,2}$ amorphous $\mathrm{Si}$ $(a-\mathrm{Si}),{ }^{3,4}$ and $\mathrm{Si}$ nanocrystallites $(\mathrm{nc}-\mathrm{Si}),{ }^{5-15}$ has been extensively investigated. In the case of $c$-Si, the nonradiative deexcitation process leads to thermal quenching of $\mathrm{Er}$ ion emission. $^{2}$ The second $a$-Si case shows a weaker temperature dependence of Er ion emission than in the $c$-Si case; however, lifetime is a problem. ${ }^{3,4}$ On the other hand, the effective excitation cross section of nc-Si is much higher than that of resonant excitation for $\mathrm{Er}$ ion in $\mathrm{SiO}_{2},{ }^{12,13}$ and the decay time of the Er ion emission is close to being temperature independent. ${ }^{11,13}$ Hence, nc-Si is regarded as one of the most promising sensitizers. More recently, we have synthesized Er-doped $\mathrm{SiO}_{2}$ including nc-Si by laser ablation and investigated the hydrogen passivation effect of interfacial defects between nc-Si and the $\mathrm{SiO}_{2}$ matrix in enhancing energy transfer from nc-Si to Er ions, resulting in enhanced Er ion emission. $^{16}$

To maximize the effectiveness of nc-Si, it is necessary to further investigate the formation process of nc-Si within an $\mathrm{SiO}_{2}$ matrix. In the present study, we investigated this by in situ annealing while taking transmission electron microscopy (TEM) measurements and ex situ annealing in an electronic furnace. The correlation between the formation of nc-Si and the Er ion was also comprehensively investigated by photo-

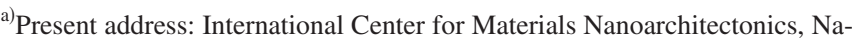
tional Institute for Materials Science, 1-1 Namiki, Tsukuba, 305-0044, Japan.

b) Also at PRESTO, Japan Science and Technology Agency, 4-1-8 Honcho Kawaguchi, Saitama 332-0012, Japan. Electronic mail FUKATA.Naoki@nims.go.jp.

c) Also at Special Research Project on Nanoscience, University of Tsukuba, Tsukuba 305-8573, Japan.
}

luminescence (PL) and electron spin resonance (ESR) measurements. The results showed the size and density of nc-Si to be important for the enhancement of Er ion emission.

\section{EXPERIMENT}

Erbium-dispersed $\mathrm{SiO}_{x}(x \leqslant 2)$ films containing nc-Si were prepared by laser ablation: Er thin films were deposited on $\mathrm{Si}$ substrates in a vacuum by ablating Er metal plates, after which the Si substrates with Er thin films were laser ablated in $\mathrm{O}_{2}$ gas at a pressure of 40 mTorr to deposit Erdispersed $\mathrm{SiO}_{x}$ films. The laser light used was a $532 \mathrm{~nm}$ Nd:YAG (YAG denotes yttrium aluminum garnet) laser light with a pulse duration of $7 \mathrm{~ns}$ and a fluence of about $4 \mathrm{~J} / \mathrm{cm}^{2}$. After the pulsed laser deposition, in situ annealing was performed to directly investigate the formation process of nc-Si in the Er-dispersed $\mathrm{SiO}_{x}$ films. This in situ annealing was performed using a TEM grid holder with a heater. After the specimens had been annealed for about $30 \mathrm{~min}$ at each temperature, TEM images were taken at four areas (an area: $40 \times 40 \mu \mathrm{m}^{2}$ ). These procedures were repeated from 200 to $900{ }^{\circ} \mathrm{C}$. To observe the effect of heating by electron beam irradiation during the TEM observations, some of the Er-dispersed $\mathrm{SiO}_{x}$ films were annealed in an electronic furnace under an Ar gas flow for $30 \mathrm{~min}$ at $400-900{ }^{\circ} \mathrm{C}$. The Er concentration was estimated to be about $2 \times 10^{20} \mathrm{~cm}^{-3}$ by Rutherford backscattering spectroscopy using $1.5 \mathrm{MeV}$ protons at a backscattering angle of $150^{\circ}$. The mean $\mathrm{Si}$ and $\mathrm{O}$ compositions of the film were also estimated to be about 40 and 60 at. $\%$, respectively.

\section{RESULTS AND DISCUSSION}

PL spectra of Er ions and nc-Si were detected using an InGaAs detector employing a lock-in amplifier system and $\mathrm{Si}$ photodiode, respectively, at RT. The excitation source was a 

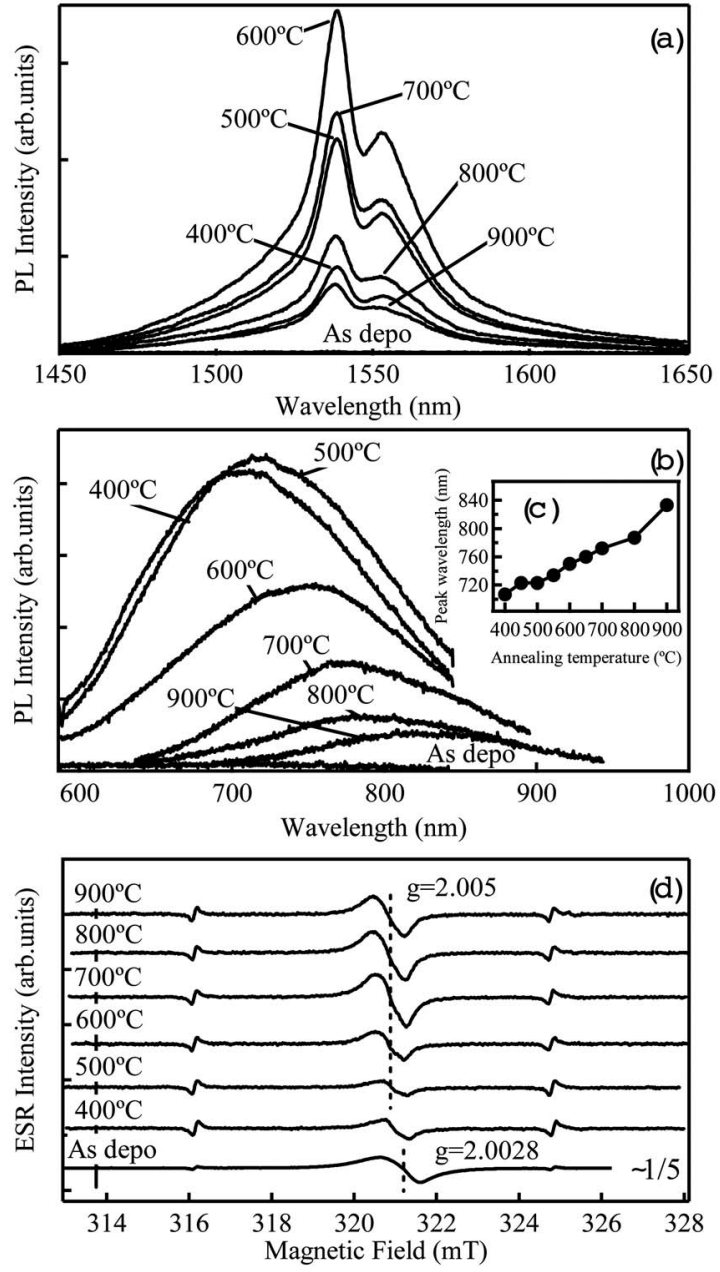

FIG. 1. Annealing temperature dependence of (a) Er PL and (b) nano-Si PL and (d) of the ESR signal of residual defects. The inset (c) shows the annealing temperature dependence of the peak position of nano-Si PL. These annealings were performed in $\mathrm{Ar}$ gas.

cw He-Cd laser light at $325 \mathrm{~nm}$. ESR measurements were carried out at RT using an $X$-band ESR spectrometer. The derivative of resonant microwave absorption with respect to the magnetic field was measured using a lock-in amplifier and magnetic field modulation with a frequency of $100 \mathrm{kHz}$. The microwave power was set at $1 \mathrm{~mW}$. An external ESR standard signal of $\mathrm{Mn}^{2+}$ in $\mathrm{MgO}$ was used to take into account possible changes in the quality factor of the microwave resonator among separate measurements. TEM (JEOL, JEM$3100 \mathrm{FEF}$ ) measurements at various temperatures were performed to make direct observations of the formation of nc-Si in the $\mathrm{SiO}_{2}$ films.

Figures 1(a) and 1(b) show the ex situ annealing temperature dependence of the PL spectra observed in two regions, and Fig. 1(d) that of the ESR signal of defects in an Er-dispersed $\mathrm{SiO}_{x}$ film. This annealing was performed in a furnace to investigate the formation of $\mathrm{nc}-\mathrm{Si}$ in Er-dispersed $\mathrm{SiO}_{x}$ films. Before the ex situ annealing, no PL spectra of Er ions or nc-Si were observed, indicating that nc-Si was not included in the film and the film was defective. Figure 1(a) shows the PL spectrum of Er ions with increasing annealing temperature. A broad PL spectrum was also observed at around $700 \mathrm{~nm}$ after annealing at $400{ }^{\circ} \mathrm{C}$; the peak showed a redshift with increasing annealing temperature, as shown in Fig. 1(b). The results are summarized in Fig. 1(c). The broad PL spectra are probably due to PL from nc-Si or amorphous Si nanograins composed of few Si atoms. ${ }^{17}$ The assignments will be explained later based on the TEM results. Hereinafter, we call these broad PL spectra nano-Si PL. The redshift can be explained by the decrease in the band gap of Si nanostructures due to the increase in the size of nc-Si or the transformation of amorphous Si nanograins to nc-Si. The intensity of the broad PL showed a maximum for the specimen annealed at $500{ }^{\circ} \mathrm{C}$. On the other hand, the PL intensity of Er ion peaked at $600{ }^{\circ} \mathrm{C}$.

The ESR signals in Fig. 1(d) are due to defects in the Er-dispersed $\mathrm{SiO}_{x}$ films. Before ex situ annealing, the $g$ value of the ESR signal was about 2.0028. The ESR signals showed a shift to a lower magnetic field with increasing annealing temperature, finally reaching a position with a $g$ value of 2.005. The $g$ values of oxygen-deficient defects $\left(\mathrm{Si}_{3-x} \mathrm{O}_{x} \equiv \mathrm{Si} \cdot ; x=1-3\right)$ in $\mathrm{SiO}_{x}$ are in the range of 2.0022.004, depending on the structures, i.e., $g$ values increase on decreasing the number of oxygen atoms. With increasing annealing temperature, the $\mathrm{SiO}_{x}$ film is transformed into perfect $\mathrm{SiO}_{2}$, resulting in the precipitation of nc-Si. Finally, defects are present only in the interface between the $\mathrm{SiO}_{2}$ matrix and nc-Si. This defect is a kind of interfacial defect, a so-called $P_{b}$ center $\left(\mathrm{Si}_{3} \equiv \mathrm{Si} \cdot\right)$. The ESR signals with $g$ value of 2.002-2.004 are thus probably due to oxygen-deficient defects in the $\mathrm{SiO}_{x}$ film and that of 2.005 due to $P_{b}$ centers. The intensity of the ESR signal increased with increasing annealing temperature. It also indicates that the formation of nc-Si proceeds with increasing annealing temperature.

TEM measurements were performed for specimens annealed in the furnace at 500, 700, and $900{ }^{\circ} \mathrm{C}$. The ex situ annealing at 500 and $700{ }^{\circ} \mathrm{C}$ showed no nc-Si formation, while the results of PL in Fig. 1(b) show the maximum intensity after ex situ annealing at $500{ }^{\circ} \mathrm{C}$. These results suggest that the annealing temperatures at 500 and $700{ }^{\circ} \mathrm{C}$ are not sufficiently high for the formation of nc-Si or that the size of $\mathrm{nc}-\mathrm{Si}$ is below $1 \mathrm{~nm}$, since the detection limit of nc-Si is around $1 \mathrm{~nm}$ in our TEM system. In the case of the latter, nc-Si with diameters below $1 \mathrm{~nm}$ is considered as the origin of the nano-Si PL, while in the former case, amorphous $\mathrm{Si}$ nanograins ${ }^{17}$ may be the origin of the PL. On the other hand, nc-Si with diameters ranging from 3 to $5 \mathrm{~nm}$ were observed in the specimen after the ex situ annealing at $900{ }^{\circ} \mathrm{C} .{ }^{16}$ The diameter distribution of nc-Si after ex situ annealing is shown in Fig. 3. The result is explained later in addition to results of in situ annealing.

To further investigate the formation process of nc-Si in the Er-dispersed $\mathrm{SiO}_{x}$ film over a wide temperature range, in situ annealing was performed during TEM observations. Typical TEM images are shown in Fig. 2. The dependences of the diameter and the density of nc-Si on the annealing temperature are summarized in Fig. 3. The data in Figs. 3(a)-3(f) correspond to in situ annealing cases, while those in Figs. 3(g) and 3(h) correspond to ex situ annealing cases. Here, the counts in the vertical axis in Fig. 3 indicate the number of nc-Si observed in a region of $6400 \mu \mathrm{m}^{2}$. The lattice fringes of Si crystal were clearly observed in the films 

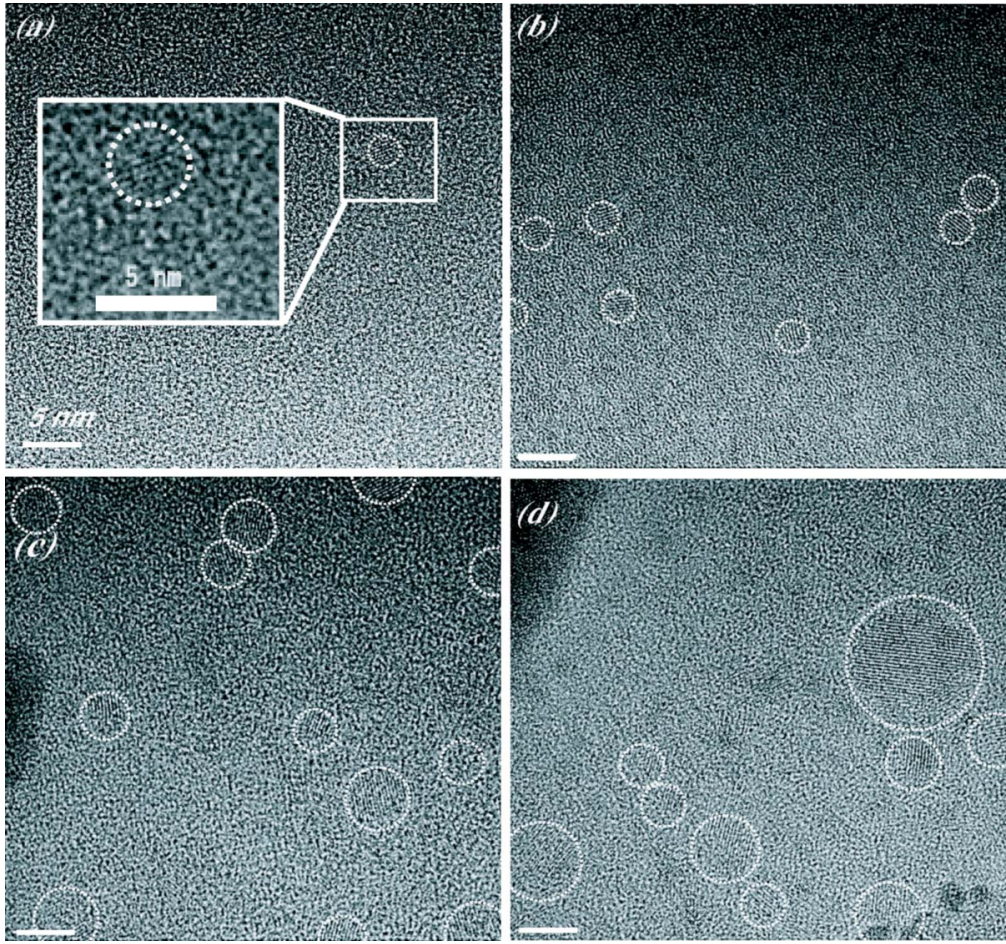

FIG. 2. (Color online) TEM micrographs of Erdispersed $\mathrm{SiO}_{x}(x \leqslant 2)$ films annealed at (a) 400, (b) 500, (c) 700, and (d) $900{ }^{\circ} \mathrm{C}$ during TEM observations. The nc-Si regions are marked by dotted circles. marked by dotted circles after in situ annealing at $400-900{ }^{\circ} \mathrm{C}$. The diameter of nc-Si was about $3 \mathrm{~nm}$ after in situ annealing at $400{ }^{\circ} \mathrm{C}$ and it reached about $10 \mathrm{~nm}$ after in situ annealing at $900{ }^{\circ} \mathrm{C}$. These results clearly show that the diameter and the density of nc-Si increase with increasing annealing temperature.

The formation temperatures of nc-Si shown in Fig. 2 are significantly different from those for the specimens annealed in the furnace. The formation of nc-Si could not be observed, even for the specimen after ex situ annealing at $700^{\circ} \mathrm{C}$, as shown in Fig. 3(g). Electron beam-assisted annealing during TEM observations is the one of the reasons, since electron beam irradiation increases the local temperature of the irradiated part of the specimen depending on the electron beam current. Considering the beam current density, the temperature rise is probably less than $300{ }^{\circ} \mathrm{C}$, indicating that another additional effect has to be considered, since the formation of nc-Si was observed from $400{ }^{\circ} \mathrm{C}$ in the case of in situ annealing, but not even for the specimen after ex situ annealing at $700{ }^{\circ} \mathrm{C}$ in the furnace. Electron-nuclear collision by electron beam irradiation may be a factor. It often induces defects, resulting in atomic displacement by the subsequent diffusion of defects. This probably promotes the precipitation of amorphous Si nanograins and finally their crystallization. The role of the electron beam-assisted annealing is to increase the temperature in the irradiated part of the specimen and to cause the precipitation of $\mathrm{nc}-\mathrm{Si}$, while that of the electron-nuclear collision causes the further enhancement of the precipitation of nc-Si by using defects introduced. The result of ex situ annealing at $900{ }^{\circ} \mathrm{C}$ in Fig. 3(h) shows the similar tendency to that for the case of in situ annealing at $500-600{ }^{\circ} \mathrm{C}$ in Figs. 3(a)-3(c). However, the density of nc-Si for the ex situ annealing case is lower than that for the in situ annealing cases. Considering these results, we think that the electron-nuclear collision corresponds to the tem-
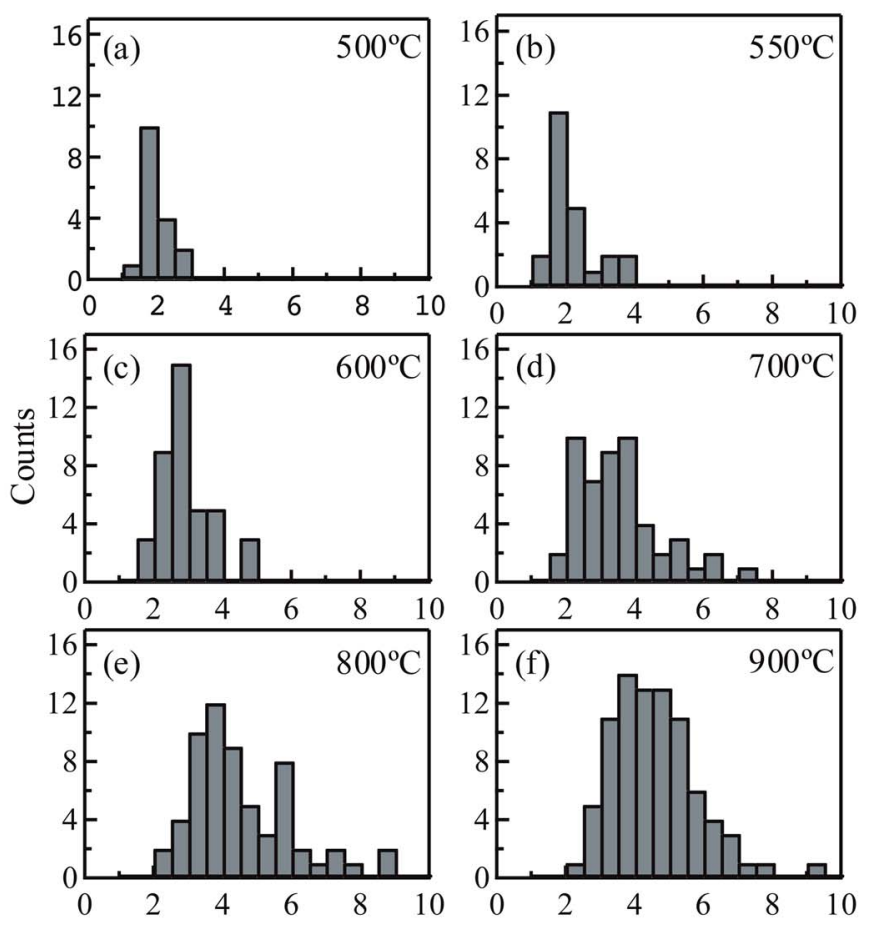

Diameter (nm)

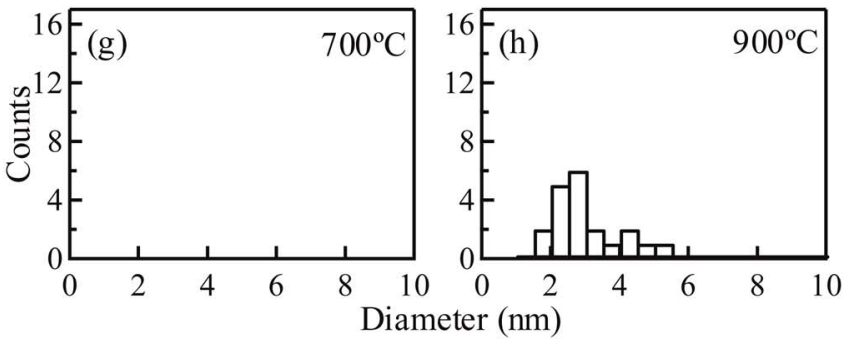

FIG. 3. (Color online) Histograms of diameter distribution of nc-Si formed in Er-dispersed $\mathrm{SiO}_{x}(x \leqslant 2)$ films by in situ annealing at (a) 500, (b) 600, (c) 700, (d) 800, and (e) $900{ }^{\circ} \mathrm{C}$ and ex situ annealing at (f) 700 and (g) $900{ }^{\circ} \mathrm{C}$. 


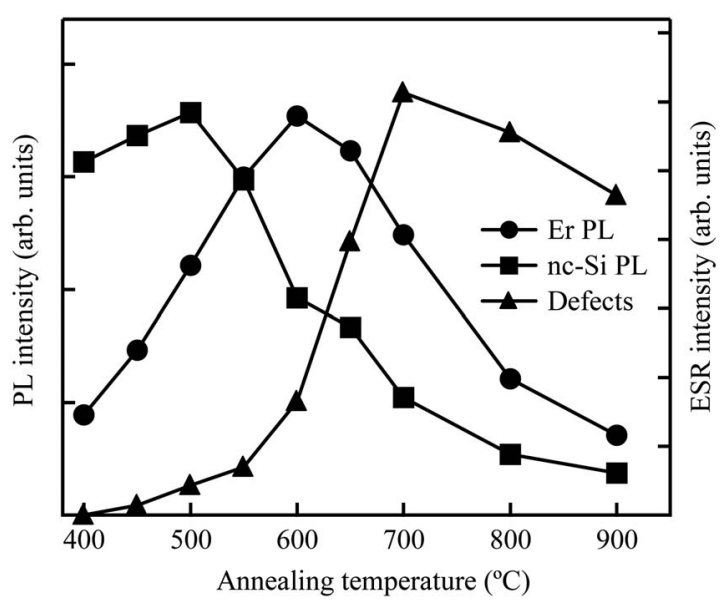

FIG. 4. Annealing temperature dependence of the PL intensities of Er ions and nano-Si, and of the ESR intensity of residual defects.

perature rise of about $100{ }^{\circ} \mathrm{C}$. We also investigated the effect on temperature during electron beam irradiation. A sample was annealed at $700{ }^{\circ} \mathrm{C}$ for $30 \mathrm{~min}$ in TEM chamber without electron beam irradiation and then investigated by TEM at RT. No trace of crystallization was observed by TEM at RT. This result indicates that irradiation temperature is important for the formation of nc-Si, namely, the electronic and collisional effects by the electron beam are thermally activated.

Figure 4 shows a summary of the results of Fig. 1, namely, the results of ex situ annealing. There is a clear inverse correlation between the intensity of nano-Si PL and that of the ESR defect signal. This means that the interfacial defects formed between nano-Si and the surrounding $\mathrm{SiO}_{x}$ matrix act as nonradiative defects. The decrease in the intensity of nano-Si PL can thus be mainly explained by the increase in the size of $\mathrm{nc}-\mathrm{Si}$, and thereby the formation of interfacial defects. There is another reason for the decrease in the intensity of nano-Si PL, namely, the energy transfer from nano-Si to Er ions. In fact, an inverse correlation between $\mathrm{Er}$ PL and nano-Si PL was also observed in the region of $500-600{ }^{\circ} \mathrm{C}$, indicating that excited nano-Si transfer energy to Er ions. Based on these, the increase in the Er PL intensity is due to the formation of nano-Si of suitable size and thereby an increase in energy transfer from nano-Si. On the other hand, the decrease is due to the decrease in energy transfer from nano-Si to Er ions, which is caused by the increase in the size of nano-Si and the formation of interfacial defects. The remaining problem concerns the suitable size of nano-Si. As already described, no formation of nc-Si was observed in the specimen annealed at below $700{ }^{\circ} \mathrm{C}$. This result shows that nc-Si with a diameter less than $1 \mathrm{~nm}$ or amorphous $\mathrm{Si}$ nanograins are effective for enhancing $\mathrm{Er}$ PL.

\section{CONCLUSION}

The formation of nc-Si in Er-dispersed $\mathrm{SiO}_{x}(x \leqslant 2)$ films was investigated by performing TEM measurements during in situ annealing. The correlation between the formation of nc-Si and Er ion emission was also comprehensively investigated by PL and ESR measurements. The results show that the formation of nano-Si region with the suitable size is important for the enhancement of Er ion emission.

\section{ACKNOWLEDGMENTS}

TEM observations were partly supported by the Nanotechnology Support Project of the Ministry of Education, Culture, Sports, Science and Technology (MEXT), Japan.

${ }^{1}$ S. Coffa, F. Priolo, G. Franzo, V. Bellani, A. Carnera, and C. Spinclla, Phys. Rev. B 48, 11782 (1993).

${ }^{2}$ F. Priolo, G. Franzò, S. Coffa, A. Polman, S. Libertino, R. Barklie, and D. Carey, J. Appl. Phys. 78, 3874 (1995).

${ }^{3}$ W. Fuhs, I. Ulber, G. Weiser, M. S. Bresler, O. B. Gusev, A. N. Kuznetsov, V. Kh. Kudoyarova, E. I. Terukov, and I. N. Yassievich, Phys. Rev. B 56, 9545 (1997).

${ }^{4}$ H. Kuhne, G. Weiser, E. I. Terukov, A. N. Kuznetsov, and V. Kh. Kudoyarova, J. Appl. Phys. 86, 896 (1999).

${ }^{5}$ J. H. Shin, G. N. van den Hoven, and A. Polman, Appl. Phys. Lett. 66, 2379 (1995)

${ }^{6}$ U. Hommerich, F. Namavar, A. Cremins, and K. L. Bray, Appl. Phys. Lett. 68, 1951 (1996).

${ }^{7}$ M. Fujii, M. Yoshida, S. Hayashi, and K. Yamamoto, J. Appl. Phys. 84, 4525 (1998).

${ }^{8}$ S. Seo and J. H. Shin, Appl. Phys. Lett. 75, 4070 (1999).

${ }^{9}$ G. Franzo, V. Vinciguerra, and F. Priolo, Appl. Phys. A A69, 3 (1999).

${ }^{10}$ M. L. Brongersma, P. G. Kik, A. Polman, K. S. Min, and H. A. Atwater, Appl. Phys. Lett. 76, 351 (2000).

${ }^{11}$ P. G. Kik, M. L. Brongersma, and A. Polman, Appl. Phys. Lett. 76, 2325 (2000).

${ }^{12}$ F. Priolo, G. Franzò, D. Pacifici, V. Vinciguerra, F. Iacona, and A. Irrera, J. Appl. Phys. 89, 264 (2001).

${ }^{13}$ A. J. Kenyon, C. E. Chryssou, C. W. Pitt, T. S. Iwayama, D. E. Hole, N. Sharma, and C. J. Humphreys, J. Appl. Phys. 91, 367 (2002).

${ }^{14} \mathrm{C}$. Li, K. Kondo, T. Makimura, and K. Murakami, Jpn. J. Appl. Phys., Part 142,3424 (2003).

${ }^{15}$ T. Makimura, K. Kondo, H. Uematsu, C. Li, and K. Murakami, Appl. Phys. Lett. 83, 5422 (2003).

${ }^{16}$ N. Fukata, C. Li, H. Morihiro, M. Mitome, Y. Bando, and K. Murakami, Appl. Phys. A: Mater. Sci. Process. 84, 395 (2006).

${ }^{17}$ F. Enrichi, G. Mattei, C. Sada, E. Trave, D. Pacifici, G. Franzo, F. Priolo, F. Iacona, M. Prassas, M. Falconieri, and E. Borsella, Opt. Mater. (Amsterdam, Neth.) 27, 904 (2005). 